\title{
Hydrogen-accelerated spontaneous microcracking in high-strength aluminium alloys
}

\author{
Tomohito Tsuru ${ }^{1,2,3^{*}}$, Kazuyuki Shimizu $\mathbb{1 0}^{4}$, Masatake Yamaguchi, ${ }^{5,2}$, Mitsuhiro Itakura ${ }^{5}$, \\ Kenichi Ebihara" ${ }^{5}$, Artenis Bendo ${ }^{6}$, Kenji Matsuda ${ }^{6}$ \& Hiroyuki Toda ${ }^{4}$
}

Aluminium alloys are re-evaluated as most feasible way to satisfy the industrial needs of light-weight structural materials. However, unlike conventional structural metals such as iron and titanium, aluminium does not have easily accessible secondary phases, which means that aluminium-based alloys cannot be strengthened by harnessing multiple phases. This leaves age hardening as the only feasible strengthening approach. Highly concentrated precipitates generated by age hardening generally play a dominant role in shaping the mechanical properties of aluminium alloys. In such precipitates, it is commonly believed that the coherent interface between the matrix and precipitate does not contribute to crack initiation and embrittlement. Here, we show that this is not the case. We report an unexpected spontaneous fracture process associated with hydrogen embrittlement. The origin of this quasi-cleavage fracture involves hydrogen partitioning, which we comprehensively investigate through experiment, theory and first-principles calculations. Despite completely coherent interface, we show that the aluminium-precipitate interface is a more preferable trap site than void, dislocation and grain boundary. The cohesivity of the interface deteriorates significantly with increasing occupancy, while hydrogen atoms are stably trapped up to an extremely high occupancy over the possible trap site. Our insights indicate that controlling the hydrogen distribution plays a key role to design further highstrength and high-toughness aluminium alloys.

The design of strong, lightweight structural materials is important for energy saving and sustainability. Although aluminium ( $\mathrm{Al}$ ) alloys are prime candidates to meet the increasing demand for lightweight materials for use in commercial applications, the fundamental approach to increasing the strength and functionality of these alloys has not been improved since age hardening of duralumin was first developed by A. Wilm in $1906^{1}$. The strength of $\mathrm{Al}$ alloys generally depends on the distribution of fine precipitates nucleated from a supersaturated solid solution in the $\mathrm{Al}$ matrix. Traditionally, Al-alloy strength is increased by adding major alloying elements $(\mathrm{Si}, \mathrm{Mg}, \mathrm{Zn}, \mathrm{Cu})$ and some minor elements $(\mathrm{Ni}, \mathrm{Si})$ that nucleate precipitates. The trade-off relationship between strength and susceptibility to hydrogen embrittlement (HE) is also recognized as a common problem with metals and high-strength $\mathrm{Al}$ alloys ${ }^{2-5}$. The hydrogen environment behaviour is still unknown because hydrogen is not likely to be soluble or to nucleate hydrides in an Al matrix as compared with other lightweight materials such as titanium 6 .

The $7 \mathrm{xxx}$ series of alloys are high-strength $\mathrm{Al}$ alloys that contain zinc $(\mathrm{Zn})$ and magnesium $(\mathrm{Mg})$ as the major alloying elements for age hardening as well as a small quantity (below $1 \mathrm{wt} \%$ ) of other elements for corrosion resistance ${ }^{7}$. In these alloys, $\mathrm{MgZn}_{2}$ precipitates are formed according to the following transformation through the ageing process: supersaturated solid solutions (SSSS) $=>\mathrm{GP}$ zone $=>\eta^{\prime}=>\eta-\mathrm{MgZn}_{2}{ }^{8,9}$. Researchers have been working on a number of efforts to reduce the hydrogen concentration as a way to prevent $\mathrm{HE}^{10}$, as the presence of a supersaturated hydrogen concentration in practical use generally enhances ductile fracture due to the high-density micropore distribution. More recently, however, a small portion of dissolved hydrogen has been found to cause an anomalous brittle fracture known as "quasi-cleavage" fracture ${ }^{11,12}$.

\footnotetext{
${ }^{1}$ Nuclear Science and Engineering Center, Japan Atomic Energy Agency, Tokai-mura, Ibaraki, 319-1195, Japan. ${ }^{2}$ Elements Strategy Initiative for Structural Materials, Kyoto University, Sakyo-ku, Kyoto, 606-8501, Japan. ${ }^{3}$ PRESTO, Japan Science and Technology Agency, Kawaguchi, Saitama, 332-0012, Japan. ${ }^{4}$ Department of Mechanical Engineering, Kyushu University, Fukuoka, Fukuoka, 819-0395, Japan. ${ }^{5}$ Center for Computational Science and e-Systems, Japan Atomic Energy Agency, Tokai-mura, Ibaraki, 319-1195, Japan. ${ }^{6}$ Graduate School of Science and Engineering for Research, University of Toyama, Toyama, Toyama, 930-8555, Japan. *email: tsuru.tomohito@jaea.go.jp
} 
In case of martensitic steel in which quasi-cleavage fracture was often observed, this type of cleavage fracture is caused by hydrogen-enhanced and plasticity-mediated decohesion at the interface ${ }^{13-15}$. In $\mathrm{Al}$ alloys, in contrast, this quasi-cleavage fracture exhibits distinctive features. The fracture behaviour cannot be explained by any other fracture mechanism in that the crack propagation occurs along the (111) plane, corresponding to the slip plane, although the plastic deformation does not mediate the crack propagation. In addition, the quasi-cleavage fracture observed in Al alloys differs from that of well-known fracture modes in metals such as hydrogen-induced cracking $^{16}$, which is generally caused by hydrogen-enhanced decohesion (HEDE) at cleavage planes and grain bound$\operatorname{aries}^{17-20}$, hydrogen-induced local plasticity (HELP) ${ }^{21-23}$, and hydrogen-mediated micropore distribution ${ }^{10,24,25}$.

Here we report on the HE mechanism related to quasi-cleavage fracture unique to Al alloys using three-dimensional observation technique and atomistic simulations. Synchrotron X-ray imaging and scanning electron microscopy (SEM) with energy dispersive X-ray spectroscopy (EDS) analysis are used to capture the characteristic features of the fracture and, in particular, to elucidate the relationship between the fracture surface and coherent surface planes of the $\eta$-phase precipitates. We focus on the hydrogen embrittlement of high-strength $\mathrm{Al}-\mathrm{Zn}-\mathrm{Mg}$ alloys and estimate the equilibrium partitioning of hydrogen in this $\mathrm{Al}$ alloys using a thermodynamic model based on first-principles density functional theory (DFT) calculations.

\section{Results and Discussion}

Experimental observation of brittle fracture mode. $\mathrm{Al}-10.0 \mathrm{Zn}-1.2 \mathrm{Mg}$ alloys were designed to investigate fracture behaviour in a hydrogen environment. In the present study, we investigated brittle fracture using following specimen. We prepared tensile specimens containing a variety of defects (vacancy, dislocation, grain boundary and precipitate) in which hydrogen diffuses to the trap sites (see the methods section for details). Figure 1 summarises the observations of the fracture surface obtained in the tensile test. The fracture surface obtained by synchrotron X-ray tomography, as shown in Fig. 1(a), clearly corresponds to brittle fracture. According to the tomography images, the crack is found to propagate gradually and undulatory along various quasi-cleavage facets at which hydrogen atoms are sufficiently trapped. Interestingly, two fracture modes were also observed in the fracture surface - intergranular fracture (IGF) and quasi-cleavage fracture (QCF) - where crack propagation tends to proceed selectively depending on the tensile direction and local stress condition around the crack tip. IGF is caused by grain boundary decohesion due to hydrogen segregation ${ }^{26,27}$.

The fracture surface of the QCF has no-dimple and no-planar morphologies and is unique because the observed fracture surface cannot be explained by any other fracture modes. With regard to the QCFs, low-voltage SEM images on this surface are shown in Fig. 1(b). Surprisingly, very dense white particle are observed on the fracture surface. The particle was identified as $\mathrm{MgZn}_{2}$ precipitate through EDS mapping, as shown in Fig. 1(c). The enlarged view of the fracture surface shown in Fig. 1(b) indicates that the fracture occurs along the Al$\mathrm{MgZn}_{2}$ precipitate interface given that almost the same number of craters as precipitates were observed on the fracture surface. The typical image of $\mathrm{Al}-\mathrm{MgZn}_{2}$ interface are preliminarily investigated by transmission electron microscopy (See Supplementary Information). This tells us that the location of craters in one fracture surface correspond to that of precipitates in the other fracture surface. These experimental observations suggest that hydrogen trapping at the $\mathrm{Al}-\mathrm{MgZn}_{2}$ precipitate interface induces decohesion and originate quasi-cleavage crack, promoting $\mathrm{HE}$ behaviour. Whether or not hydrogen atoms preferably segregate into the $\mathrm{Al}-\mathrm{MgZn}_{2}$ precipitate interface depends on the magnitude correlation of the binding energy between hydrogen and each defect structure. We thus evaluated the tendency of hydrogen trapping at various sites using first-principles calculations.

Binding energy of hydrogen at various trap sites. It is well known that the $\mathrm{Al}-\mathrm{MgZn}_{2}$ interface, which plays enhancing the strength of alloys, does not influence the degradation of fracture behaviour in terms of interfacial decohesion because the $\eta$-phase $\mathrm{MgZn}_{2}$ precipitate nucleates the completely coherent interface with the $\mathrm{Al}$ (111) plane. This kind of interface generally does not tend to absorb interstitial atoms since the coherent interface contributes little to the increase in free volume. However, the fracture surface of the QCF mode indicates decohesion at the $\mathrm{Al}-\mathrm{MgZn}_{2}$ interface as a result of hydrogen segregation.

We comprehensively investigated the binding energy between hydrogen and various defect structures such as vacancy, edge/screw dislocations, grain boundary, and $\eta-\mathrm{MgZn}_{2}$ precipitate. The results are shown in Fig. 2(a). Atomic models of defect structures for DFT calculations at which hydrogen atoms are to be trapped are given in the same figure. The computational setup for the DFT calculations is described in the methods section. The most stable interface is expected to be reached by minimizing the elastic strain energy associated with the difference in the lattice constants between the $\mathrm{Al}$ matrix and $\mathrm{MgZn}_{2}$, and is found to be the $\eta 2$ interface, which is consistent with the high-resolution transmission electron microscopy (HRTEM) observations ${ }^{28,29}$.

As a result, the $\eta 2$ interface was taken as the target interface, where the $\eta 2$ interface has a coincidence axis along $\mathrm{Al}[1 \overline{1} 0] / / \mathrm{MgZn}_{2}[\overline{1} 100]$ and $\mathrm{AlAl}[\overline{1} 12] / / \mathrm{MgZn}_{2}[11 \overline{2} 0]$ normal to the interface $\left(\mathrm{Al}(111) / / \mathrm{MgZn}_{2}(0001)^{8}\right.$. The binding energies with all trap sites are summarized in Fig. 2(b), where the binding energies are defined as the difference in the energy between defects and stable tetrahedral sites in bulk Al. According to our calculations, hydrogen atoms are definitely trapped at the grain boundary through internal diffusion ${ }^{30}$. Both edge and screw dislocations do not have a significant influence on hydrogen trapping, while hydrogen changes the edge/screw character of dislocations and slip planarity ${ }^{31-33}$. On the other hand, the binding energy of the inner region of the $\mathrm{MgZn}_{2}$ crystal is extremely small (the biding energy at most stable site is $0.079 \mathrm{eV}$ ), which indicates that hydrogen tends not to be absorbed into the precipitate due to the unstable sites in $\mathrm{MgZn}_{2}{ }^{34}$. Interestingly, the results show that the binding energy at the $\mathrm{Al}-\mathrm{MgZn}_{2}$ interface is higher than that of the grain boundary and vacancy ${ }^{30,34}$, even if the interface remains completely coherent (no lattice mismatch) and the free volume around the interface is small. The Al- $\mathrm{MgZn}_{2}$ interface is therefore one of the most favourable trap sites among possible defect structures in $\mathrm{Al}$ alloys such as vacancy, edge/screw dislocation and grain boundaries. Incidentally, the significant stability of 
a
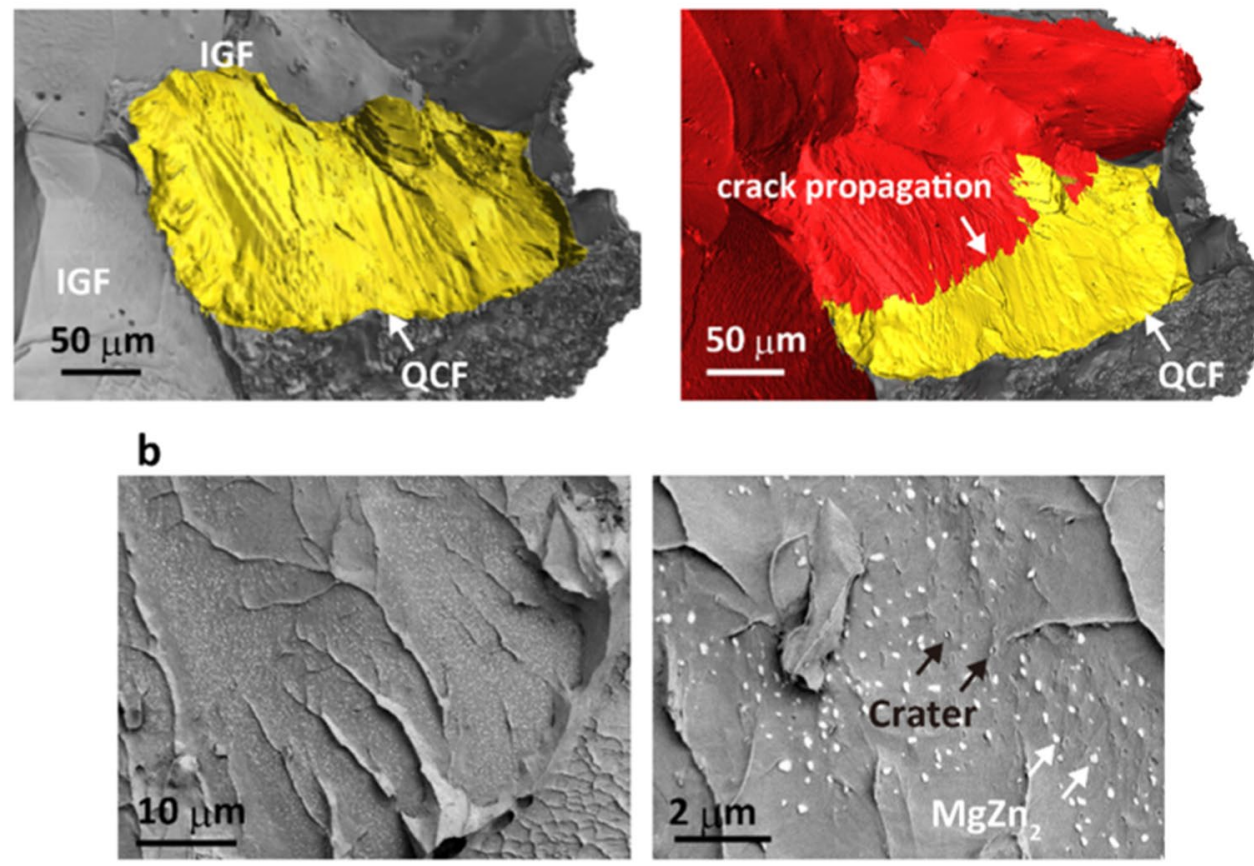

C
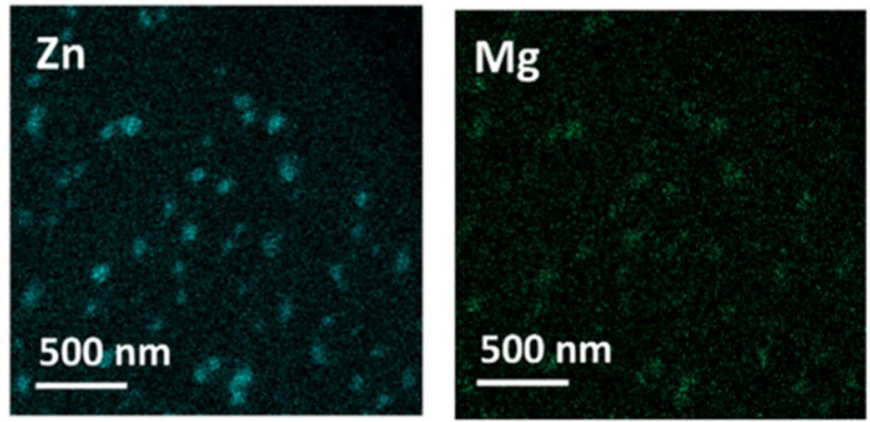

Figure 1. Experimental observation of the fracture surface of an Al-Zn-Mg alloy. (a) Three-dimensional tomographic images of fractured $\mathrm{Al}-\mathrm{Zn}-\mathrm{Mg}$ alloy samples captured by synchrotron X-ray tomography. Two fracture modes - intergranular fracture (IGF) and quasi-cleavage fracture (QCF) - can be observed on the fracture surface. Crack propagation can be also captured by tomographic image on the right, where the image was obrained at $\varepsilon=11 \%$ (near $\tau_{\mathrm{UTS}}$ ). The crack is found to propagate gradually and undulatory along various quasi-cleavage facets at which hydrogen atoms are sufficiently trapped. (b) Low-voltage SEM images of this fracture surface, in which very dense white and black dots correspond to an $\mathrm{MgZn}_{2}$ precipitate and the crater of the precipitate, respectively. (c) EDS mapping of $\mathrm{Zn}$ and $\mathrm{Mg}$ on the fracture surface, indicating a clear region of precipitate.

the pore region, including of the surface and $\mathrm{H}_{2}$ molecule, implies that hydrogen segregation tends to occur spontaneously, stabilizing the surface and forming $\mathrm{H}_{2}$ molecules in spite of the highly concentrated hydrogen around defects.

Hydrogen partitioning. As it is still difficult to examine experimentally how hydrogen atoms are actually distribute in the interior area of materials, we implemented the thermodynamic approach incorporated with atomistic simulations. The HE behaviour is dominated by hydrogen partitioning. The equilibrium states of hydrogen partitioning can be expressed as $C_{\text {tot }}=\theta_{L} N_{L}+\sum \theta_{i} N_{i}+C_{\text {pore }}$, where $C_{\text {tot }}$ is the total hydrogen content in an Al alloy, and $\theta$ and $N$ represent the occupancy and the trap site density for the interstitial site $L$ and the $i$ th trap site, respectively. We evaluated the binding energies of hydrogen at various trap sites using DFT calculations, and evaluated the site occupancy directly using the expression for equilibrium segregation: $\theta_{i} /\left(1-\theta_{i}\right)=\theta_{L} \exp \left(E_{i} / R T\right)$, where $R$ is the molar gas constant, $T$ is the temperature, and $E_{i}$ corresponds to the binding energy of various trap sites evaluated by DFT calculations in Fig. 2(b). The theoretical estimate of site occupancy is given in Fig. 3(a). Site occupancy differs widely, reflecting the exponential contribution of the binding energies.

Next, we estimated the actual trap site densities directly by means of experimental observation. The area of the grain boundary, dislocation density, pore morphology, vacancy concentration, and the area of the 
a

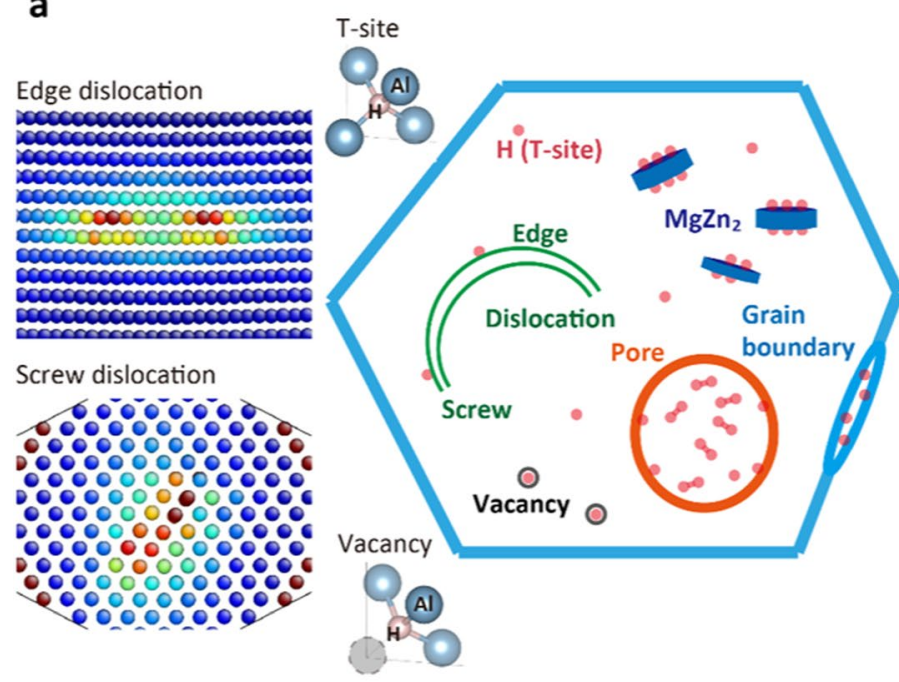

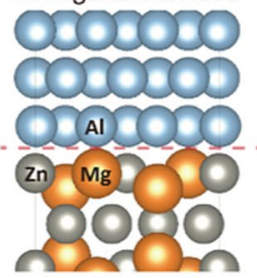

Grain boundary

(e.g. $\Sigma 5(012))$

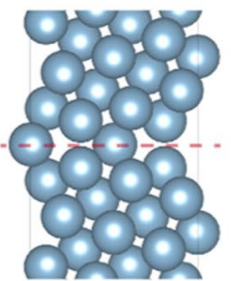

b

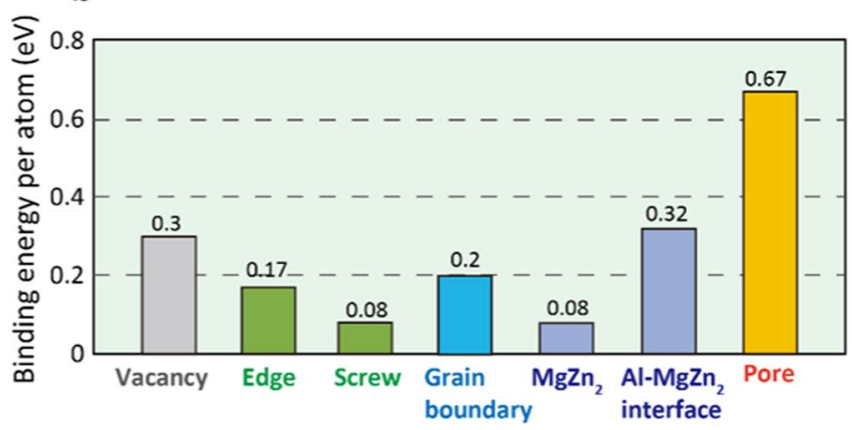

Type of defect structure as a trap site

Figure 2. Schematic image of possible trap sites of hydrogen and their binding energy. (a) Schematic showing various defect structures as trap sites of hydrogen in $\mathrm{Al}$ alloys. Atomic models of these defect structures used for DFT calculations, where T-site corresponds tetragonal site. (b) Binding energy per atom between hydrogen and defect structures such as vacancy, edge/screw dislocations, grain boundary, and $\eta-\mathrm{MgZn}_{2}$ precipitate evaluated by first-principles calculations.

precipitate interface were evaluated by experimental techniques and mathematical models (see Supplementary Fig. S1). Total grain boundary area per unit aluminium volume was calculated based on grain size. The dislocation densities were evaluated using the Williamson-Hall method ${ }^{35}$. The detailed procedure to estimate dislocation density was explained in our previous study ${ }^{36}$ and the value was estimated to be $4.07 \times 10^{14} \mathrm{~m}^{-231}$. The volume and surface area of all the pores present in the gauge region of the prepared alloys were analysed using the marching cubes algorithm ${ }^{37}$, where the three-dimensional morphology of pores can be measured by synchrotron X-ray tomography. The area of the $\eta-\mathrm{MgZn}_{2}$ precipitate interface was calculated from TEM observations ${ }^{29}$. The diameter and height of the $\eta-\mathrm{MgZn}_{2}$ precipitate were observed to be approximately $20 \mathrm{~nm}$ and $5 \mathrm{~nm}$, respectively.

Note that the trap site density needs to be measured for each sample since it differs depending on the conditions of both the deformation processing and heat treatment. Accordingly, we can estimate hydrogen partitioning at various defect sites by taking all contributions of the binding energy and trap site density for various sites, as shown in Fig. 3(b), where each trapped hydrogen content, $C_{i}$, is given by $\theta_{i} N_{i}$. Our estimation indicates that hydrogen atoms are trapped prominently at the $\mathrm{Al}-\mathrm{MgZn}_{2}$ interface due to the high presence of $\mathrm{MgZn}_{2}$ precipitates, while the occupancy of the pore region itself is highest according to the result of the binding energy. Surprisingly, hydrogen atoms tend to be trapped at the interface more than at the grain boundary. The interfacial cohesive energy of the coherent $\mathrm{Al}-\mathrm{MgZn}_{2}$ interface is sufficiently high but slightly smaller than that of the grain boundaries (see Supplementary Information); therefore hydrogen partitioning has a dominant influence on both intergranular and quasi-cleavage factures through $\mathrm{HE}$.

Spontaneous hydrogen-accumulated cleavage. DFT calculations were again carried out to explore the relationship between cohesive energy and occupancy at the interface and confirm whether such highly-concentrated hydrogen is actually possible at the the Al- $\mathrm{MgZn}_{2}$ interface. The binding energy per atom in terms of occupancy is shown in Fig. 4(a). Stable configurations of hydrogen at the interface associated with specific occupancies are given in Fig. 4(b). Despite the case of a coherent interface, the binding energy does not 
a

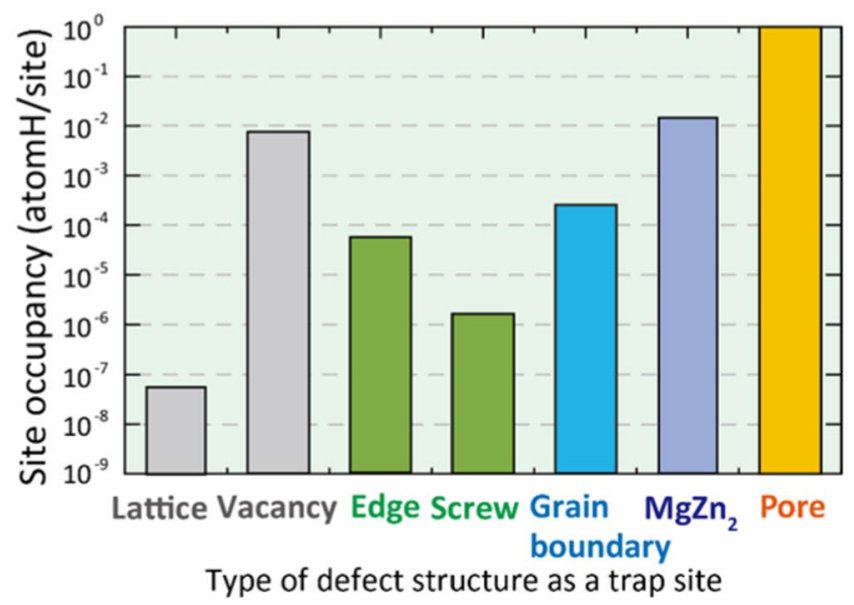

b

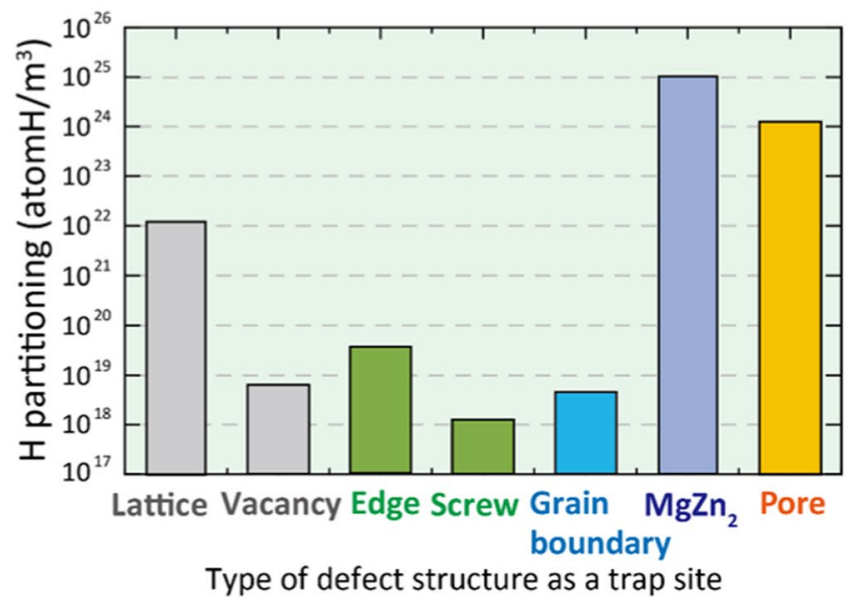

Figure 3. Hydrogen partitioning behaviour in Al-Zn-Mg alloys. Theoretically-derived estimates of (a) H occupancy and (b) hydrogen partitioning at various defect sites including all contributions of the binding energy shown in Fig. 2(b) and the trap site density for various sites. Site occupancy and actual concentration were derived based on thermodynamic equilibrium condition and first-principles calculations.

decrease even if the occupancy increases up to the maximum occupancy (ten hydrogen atoms on the interface of the present unit cell). The trap efficiency is still higher than that of other defect structures, such as grain boundaries. In the case that the occupancy exceeds the limit, hydrogen atoms tend to stably nucleate as hydrogen molecules, as shown in Fig. 4(b). As a result, the binding energy continues to increase.

The interfacial cohesive energy corresponding to the occupancy is also given in Fig. 4(a). We found that the cohesive energy decreases as the occupancy increases. These results show that the cohesivity of the interface deteriorates significantly with increasing occupancy, while hydrogen atoms are stably trapped one after the other. As a result, the QCF is induced spontaneously at the $\mathrm{Al}-\mathrm{MgZn}_{2}$ interface when there is a high concentration of hydrogen atoms. Macroscopic crack propagation was subsequently enhanced as the QCF caused by hydrogen trapping is more active which provides easy crack propagation path related to the direction of the applied stress. This hydrogen-accelerated QCF is unique to the $\mathrm{Al}-\mathrm{MgZn}_{2}$ interface in $\mathrm{Al}$ alloys; it does not occur in Fe alloys because hydrogen is not trapped as significantly at the interface within them ${ }^{38}$. The surface energy of $\mathrm{Al}$ is intrinsically lower than that of transition metals ${ }^{30}$ and, moreover, hydrogen is very stable at the surface, which decreases the surface energy. Our findings reveal that hydrogen atoms tend to segregate at the $\mathrm{Al}-\mathrm{MgZn} \mathrm{interface} \mathrm{rather}_{2}$ than at grain boundaries and promote hydrogen embrittlement at the interface, resulting in spontaneous microcracking. We believe that to suppress the intensive segregation at the $\mathrm{Al}-\mathrm{MgZn}_{2}$ leads to design high-toughness $\mathrm{Al}$ alloys.

In conclusion, we have discovered a new hydrogen-induced embrittlement mechanism that underpins quasi-cleavage fracture in $\mathrm{Al}$ alloys. Despite the coherent atomic configuration, the $\mathrm{Al}-\mathrm{MgZn}_{2}$ interface is a more preferable hydrogen trap site than other defects in terms of binding energy and hydrogen partitioning. The cohesivity of the interface deteriorates significantly with increasing hydrogen occupancy, while hydrogen atoms are stably trapped up to an extremely high occupancy that is equivalent to spontaneous cleavage. These new findings provide a strategy to design high-toughness $\mathrm{Al}$ alloys to suppress $\mathrm{HE}$ behaviour in high-strength $\mathrm{Al}$ alloys. 


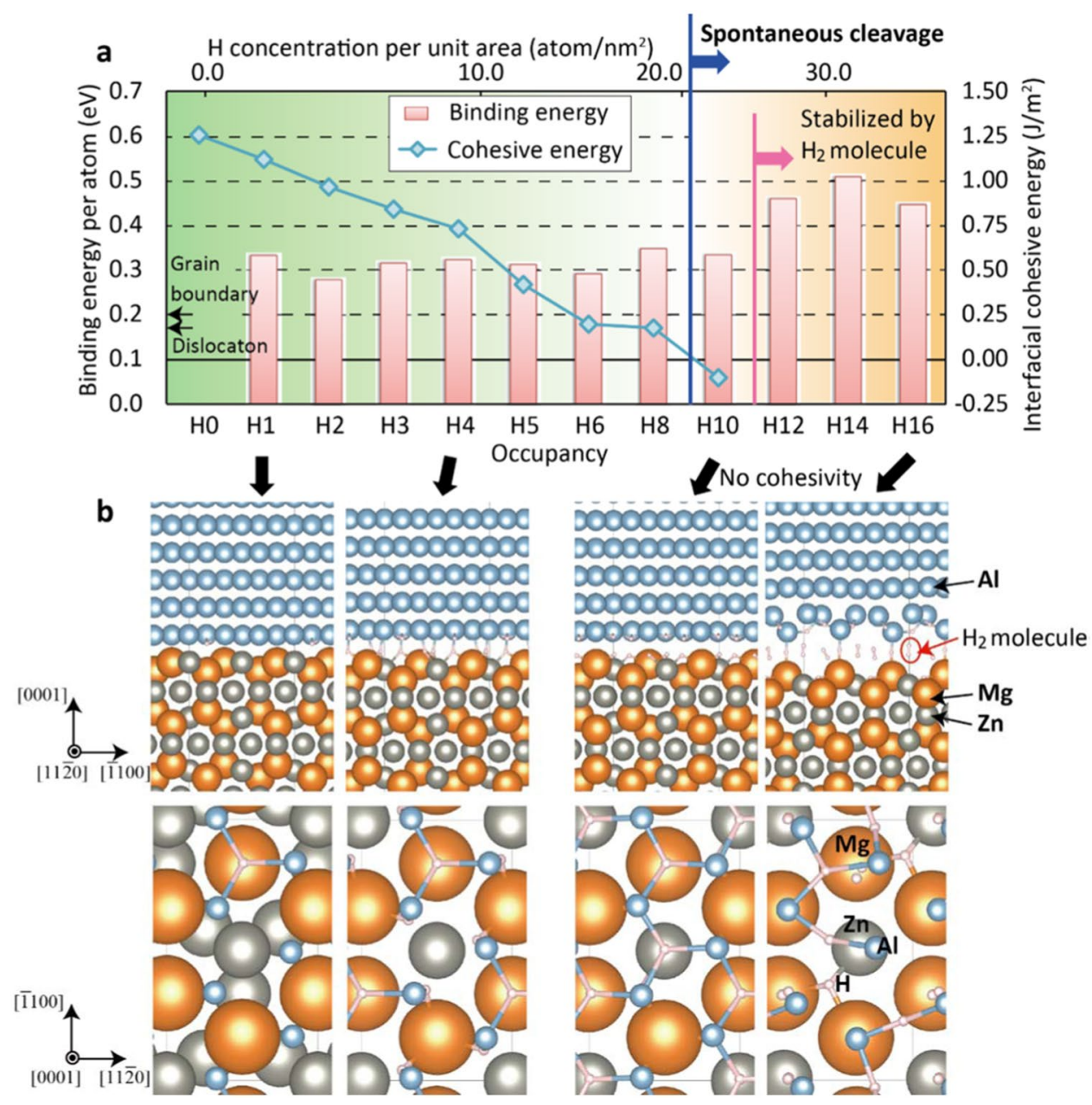

Figure 4. Mechanism of spontaneous cleavage induced by hydrogen segregation at the $\mathrm{Al}-\mathrm{MgZn} \mathrm{n}_{2}$ interface. (a) Binding energy per atom and interfacial cohesive energy as a function of occupancy. (b) The most stable configurations of hydrogen at the interface associated with specific occupancies. Spontaneous cleavage occurs when the concentration of hydrogen atom reaches $22.7 \mathrm{H}$ atoms $/ \mathrm{nm}^{2}$ according to negative value of the cohesive energy in (a). Additionally, the cleavage surface becomes more stable by forming $\mathrm{H}_{2}$ molecules at the fracture surface while maintaining hydrogen segregation and spontaneous cleavage.

\begin{abstract}
Methods
Specimen preparation with hydrogen charge. First, Al-10.1Zn-1.2Mg alloys were prepared by homogenization at $773 \mathrm{~K}$ for $7.2 \mathrm{ks}$ after casting and hot rolling at $723 \mathrm{~K}$ with a rolling reduction of $50 \%$. Alloys after solution treatment were quenched in ice water and immediately subjected to ageing within 10 minutes from quenching. The sample used for the measurement of tomography and EDS maps is prepared at $393 \mathrm{~K}$ for 114 $\mathrm{ks}$ and $543 \mathrm{~K}$ for $25.2 \mathrm{ks}$ aging condition. Specimen preparation and hydrogen charging were performed using electrical discharge machining (EDM, BA8, Mitsubishi Electric, Inc.). The shape of specimens is the same as that reported in the literature ${ }^{39}$, having $0.7 \mathrm{~mm}$ in gauge length and $0.6 \times 0.6 \mathrm{~mm}^{2}$ in cross-sectional area. We confirmed the hydrogen content of the alloy using the vacuum fusion method with EDM, finding that it increased from 0.14 to 6.97 mass ppm in water purified by ion exchange resins ${ }^{31}$. The hydrogen content in the prepared $\mathrm{Al}-\mathrm{Zn}$-Mg alloys after EDM cutting by a gas chromatography-type thermal desorption analyser (PDHA-1000, NISSHA FIS, Inc.) was 6.98 mass ppm, which is almost the same as that found elsewhere $(6.97 \text { mass ppm })^{11}$. The hydrogen in specimens after EDM was presumed to be highly concentrated on the surface region. To distribute hydrogen to the interior of the specimens, the specimens were stored in acetone for approximately four days. Considering the diffusion coefficient of hydrogen in aluminium at $300 \mathrm{~K}^{40}$, the diffusion distance after four days is estimated to be $2.8 \mathrm{~mm}$, which is enough to diffuse to the inner region of the specimens.
\end{abstract}

Microstructural characterization under tensile deformation. The deformation and fracture behaviour of hydrogen-charged Al-10.1Zn-1.2Mg alloys was observed by in-situ synchrotron X-ray tomography on BL20XU of Spring-8, Japan. The methodology adopted for tomographic observation and the in-situ tensile test follow that reported elsewhere; ${ }^{11}$ a monochromatic $20 \mathrm{keV} \mathrm{X}$-ray was used to perform the tomography. The initial strain rate was set at $3 \times 10^{-3} \mathrm{~s}^{-1}$ by displacement control, and the test was performed at room temperature. Tomographic observations were taken under several conditions: no loading, near the elastic limit, 
and every approximately $3 \%$ of applied strains until the specimen fractured. The acquired X-ray datasets were three-dimensionally reconstructed by convolutional back-projection ${ }^{41}$. Following tomography, the fracture surface was observed using SEM with 1-kV acceleration voltage. Compositional analysis was performed by EDS at an accelerating voltage of $5 \mathrm{kV}$ in order to observe the precipitates on the quasi-cleavage facets.

Atomic structure of the Al-MgZn2 interface. The $\mathrm{MgZn}_{2}$ precipitate has a $\mathrm{C} 14$ Laves phase (space group P63/mmc) in its most stable state ${ }^{42}$. An atomic model of an Al defect-free crystal was constructed with a lattice constant of $4.04 \AA$. The interstitial site for hydrogen segregation was then chosen as the centre of the tetrahedron in the Al. Coherent interfacial models were subsequently constructed by finding the minimum number of unit cells of both $\mathrm{MgZn}_{2}$ and $\mathrm{Al}$ that are necessary to minimize the elastic strain along the direction parallel to the interface ${ }^{34}$.

The number of unit cells was $\operatorname{MgZn}_{2}[\overline{1} 100]: \operatorname{Al}[1 \overline{1} 0]=1: 3$ and $\operatorname{MgZn}_{2}[11 \overline{2} 0]: \operatorname{Al}[\overline{1} 12]=1: 1$ for $\eta 2-\operatorname{MgZn}_{2}$, where the $\eta 2$ interface could be composed of the smallest multiple numbers of unit cells. The widths of the Al and $\mathrm{MgZn}_{2}$ regions along the [0001] and [111] directions were determined so that the length of the multiplied unit cell was larger than $15 \AA$. We performed preliminary DFT calculations to determine the stable configuration for the other degree of freedom, i.e., the parallel shift on the interface. A vacuum layer with a width of more than $15 \AA$ was inserted, and thus all possible initial configurations were prepared. The Voronoi polyhedral technique was applied to systematically extract possible interstitial sites at the interface using voro ++ software ${ }^{43}$.

DFT calculations for hydrogen trapping. First-principles calculations were conducted within the DFT framework using the Vienna ab initio simulation package (VASP 5.2) ${ }^{44,45}$ with the Perdew-Burke-Ernzerhof generalized gradient approximation exchange-correlation density functional ${ }^{46}$. The Brillouin-zone gamma-centred $k$-point samplings were chosen using the Monkhorst-Pack algorithm ${ }^{47}$, where a $5 \times 9 \times 1 \mathrm{k}$-point was chosen for the interface model. A cut-off in plane-wave energy of $400 \mathrm{eV}$ was applied using a first-order Methfessel-Paxton scheme that employed a smearing parameter of $0.1 \mathrm{eV}$. The total energy was converged within $10^{-6} \mathrm{eV} /$ atom for all calculations. The relaxed configurations were obtained using the conjugate gradient method that terminated the search when the force on all atoms was reduced to $0.01 \mathrm{eV} / \AA$. The zero-point energy of hydrogen atoms was taken into account for the total energy. Atomic configurations were visualized using VESTA 3.4 ${ }^{48}$ and Atomeye $\mathrm{e}^{49}$ software.

Received: 30 October 2019; Accepted: 21 January 2020;

Published online: 06 April 2020

\section{References}

1. Haas, M. H. W. das Duralumin erfunden wurde. Aluminium 18, 366-367 (1936).

2. Nguyen, D., Thompson, A. W. \& Bernstein, I. M. Microstructural effects on hydrogen embrittlement in a high purity 7075 aluminium alloy. Acta Metall. 35, 2417-2425 (1987).

3. Lynch, S. P. Environmentally assisted cracking: Overview of evidence for an adsorption-induced localised-slip process. Acta Metall. 36, 2639-2661 (1988).

4. Su, H. et al. Influence of hydrogen on strain localization and fracture behavior in Al-Zn-Mg-Cu aluminium alloys. Acta Mater. 159, 332-343 (2018).

5. Yin, S. et al. Hydrogen embrittlement in metallic nanowires. Nat. Commun. 10, 2004 (2019).

6. Fast, J. D. Interaction of Metals and Gases: Thermodynamics and phase relations. Vol. 1, Philips Technical Library, (1965).

7. Schneider, R., Heine, B. \& Grant, R. J. Light metal alloys applications, in: W.A. Monteiro (Ed.), (Chapter 3) (2014).

8. Gjønnes, J. \& Simensen, C. J. An electron microscope investigation of the microstructure in an aluminium-zinc-magnesium alloys. Acta Metall. 18, 881-890 (1970).

9. Berg, L. K. et al. GP-zones in Al-Zn-Mg alloys and their role in artificial aging. Acta Mater. 49, 3443-3451 (2001).

10. Toda, H., Oogo, H., Uesugi, K. \& Kobayashi, M. Roles of Pre-Existing Hydrogen Micropores on Ductile Fracture. Mater. Trans. 50, 2285-2290 (2009).

11. Bhuiyan, M. S. et al. Influences of hydrogen on deformation and fracture behaviors of high Zn 7XXX aluminium alloys. Int. J. Fract. 200, 13-29(2016).

12. Bhuiyan, M. S. et al. The Role of Hydrogen on the Local Fracture Toughness Properties of 7XXX Aluminium Alloys. Metall. Mater. Trans. A 49, 5368-5381 (2018).

13. Gao, M. \& Wei, R. P. Quasi-cleavage and martensite habit plane. Acta Metall. 32, 2115-2124 (1984).

14. Nagao, A., Smith, C. D., Dadfarnia, M., Sofronis, P. \& Robertson, I. M. The role of hydrogen in hydrogen embrittlement fracture of lath martensitic steel. Acta Mater. 60, 5182-5189 (2012).

15. Nagao, A., Smith, C. D., Dadfarnia, M., Sofronis, P. \& Robertson, I. M. Interpretation of hydrogen-induced fracture surface morphologies for lath martensitic steel. Proc. Mater. Sci. 3, 1700-1705 (2014).

16. Robertson, I. M. et al. Hydrogen embrittlement understood. Metall. Mater. Trans. A 46, 2323-2341 (2015).

17. Pfeil, L. B. The effect of occluded hydrogen on the tensile strength of iron. Proc. Roy. Soc. (London) A 122, 128-195 (1926).

18. Troiano, A. R. The role of hydrogen and other interstitials in the mechanical behavior of metals. Trans. ASM 52, 54-80 (1960),

19. Oriani, R. A. \& Josephic, P. H. Equilibrium aspects of hydrogen-induced cracking of steels. Acta Metall. 22, 1065-1074 (1974).

20. McMahon, C. J. Jr. Hydrogen-induced intergranular fracture of steels. Eng. Fract. Mech. 68, 773-788 (2001).

21. Birnbaum, H. K. \& Sofronis, P. Hydrogen-enhanced localized plasticity-a mechanism for hydrogen-related fracture. Mater. Sci. Eng. A 176, 191-202 (1994).

22. Beacham., C. D. A new model for hydrogen-assisted cracking (hydrogen "embrittlement"). Metall. Mater. Trans. B 3, 441-455 (1972).

23. Robertson, I. M. The effect of hydrogen on dislocation dynamics. Eng. Fract. Mech. 64, 649-673 (1999).

24. Toda, H. et al. Effects of Hydrogen Micro Pores on Mechanical Properties in A2024 Aluminium Alloys. Maters. Trans. 54, 2195-2201 (2013).

25. Toda, H. et al. The True Origin of Ductile Fracture in Aluminium Alloys. Metall. Mater. Trans. A 45, 765-776 (2014).

26. Yamaguchi, M. et al. First-principles calculation of multiple hydrogen segregation along aluminum grain boundaries. Comput. Mater. Sci. 156, 368-375 (2019). 
27. Birbilis, N. \& Hinton, B. 19 - Corrosion and corrosion protection of aluminium, Fundamentals of Aluminium Metallurgy, 574-604 (2011).

28. Marioara, C. D., Lefebvre, W., Andersen, S. J. \& Friis, J. Atomic structure of hardening precipitates in an Al-Mg-Zn-Cu alloy determined by HAADF-STEM and first-principles calculations: relation to $\eta-M g Z n 2$. J. Mater. Sci. 48, 3638-3651 (2013).

29. Bendo, A. et al. Microstructure evolution in a hydrogen charged and aged $\mathrm{Al}-\mathrm{Zn}-\mathrm{Mg}$ alloy. Materialia 3, 50-56 (2018).

30. Yamaguchi, M. et al. First-principles calculation of multiple hydrogen segregation along aluminium grain boundaries. Comput. Mater. Sci. 156, 368-375 (2019).

31. Shimizu, K. et al. Hydrogen partitioning behavior and related hydrogen embrittlement in Al-Zn-Mg alloys. Eng. Frac. Mech. 216, 106503 (2019).

32. Ferreira, P. J., Robertson, I. M. \& Birndum, H. K. Hydrogen effects on the character of dislocations in high-purity aluminium. Acta Mater. 47, 2991-2998 (1999).

33. Lu, G., Zhang, Q., Kioussis, N. \& Kaxiras, E. Hydrogen-Enhanced Local Plasticity in Aluminium: An Ab Initio Study. Phys. Rev. Lett. 87, 095501 (2001).

34. Tsuru, T. et al. First-principles study of hydrogen segregation at the MgZn2 precipitate in Al-Mg-Zn alloys. Comput. Mater. Sci. 148, 301-306 (2018).

35. Ungár, T. \& Borbély, A. The effect of dislocation contrast on x-ray line broadening: A new approach to line profile analysis. Appl. Phys. Lett. 69, 173-175 (1996).

36. Bhuiyan, M. S. et al. Combined microtomography, thermal desorption spectroscopy, X-ray diffraction study of hydrogen trapping behavior in 7XXX aluminum alloys. Mater. Sci. Eng. A 655, 221-228 (2016).

37. Lorensen, W. E. \& Cline, H. E. Marching cubes: A high resolution 3D surface construction algorithm, Proc. 14th Annu. Conf. Comput. Graph. Interact. Tech. SIGGRAPH '87, vol. 21, (New York, USA: ACM Press, pp. 163-169, 1987).

38. Yamaguchi, M., Kameda, J., Ebihara, K., Itakura, M. \& Kaburaki, H. Mobile effect of hydrogen on intergranular decohesion of iron: first-principles calculations. Philos. Mag. 92, 1349-1368 (2012).

39. Su, H. et al. Assessment of hydrogen embrittlement via image-based techniques in $\mathrm{Al}-\mathrm{Zn}-\mathrm{Mg}-\mathrm{Cu}$ aluminum alloys. Acta Mater. 1, 96-108 (2019).

40. Young, G. A. \& Scully, J. R. The diffusion and trapping of hydrogen in high purity aluminium. Acta Mater. 46, 6337-6349 (1998).

41. Kak, A. C. \& Slaney, M. Principles of computerized tomographic imaging. Society for Industrial and Applied Mathematics, (2001).

42. Steurer, W. \& Dshemuchadse, J. Intermetallics, Structures, Properties, and Statistics, Oxford University Press, International Union of Crystallography Monographs on Crystallography, (2016).

43. Rycroft, C. H. V. O. R. O. + A three-dimensional Voronoi cell library in C++. CHAOS 19, 041111 (2009).

44. Kresse, G. \& Hafner, J. Ab initio molecular dynamics for liquid metals. Phys. Rev. B 47, 558-561 (1993).

45. Kresse, G. \& Furthmuller, J. Efficient iterative schemes for ab initio total-energy calculations using a plane-wave basis set. Phys. Rev. B 54, 11169-11186 (1996).

46. Perdew, J. P., Burke, K. \& Ernzerhof, M. Generalized Gradient Approximation Made Simple. Phys. Rev. Lett. 77, 3865-3868 (1996). 47. Monkhorst, H. J. \& Pack, J. D. Special points for Brillouin-zone integrations. Phys. Rev. B 13, 5188-5192 (1976).

48. Momma, K. \& Izumi, F. VESTA 3 for three-dimensional visualization of crystal, volumetric and morphology data. J. Appl. Crystallogr. 44, 1272-1276 (2011).

49. Li, J. AtomEye: an efficient atomistic configuration viewer. Model. Simul. Mater. Sci. Eng. 11, 173-177 (2003).

\section{Acknowledgements}

This work was supported by a JST Collaborative Research Based on Industrial Demand "Heterogeneous Structure Control: Towards Innovative Development of Metallic Structural Materials (Project 20100114)." T.T acknowledges the support of JSPS KAKENHI (Grant Numbers JP19K04993, JP18H05453, JP17H03409) and JST PRESTO Grant Number JPMJPR1998, Japan. Simulations were performed on the large-scale parallel computer system with SGI ICE X at JAEA.

\section{Author contributions}

H.T. coordinated the overall project. K.S., A.B. and K.M. prepared samples and carried out the main experiments. T.T., Y.M. and M.I. implemented the DFT calculations for defect structures and analyzed the data. K.S. and K.E. performed the partitioning analysis. T.T. edited the manuscript. All authors contributed to the discussion of the results, and commented on the manuscript.

\section{Competing interests}

The authors declare no competing interests.

\section{Additional information \\ Supplementary information is available for this paper at https://doi.org/10.1038/s41598-020-58834-6.}

Correspondence and requests for materials should be addressed to T.T.

Reprints and permissions information is available at www.nature.com/reprints.

Publisher's note Springer Nature remains neutral with regard to jurisdictional claims in published maps and institutional affiliations.

(c) (i) Open Access This article is licensed under a Creative Commons Attribution 4.0 International License, which permits use, sharing, adaptation, distribution and reproduction in any medium or format, as long as you give appropriate credit to the original author(s) and the source, provide a link to the Creative Commons license, and indicate if changes were made. The images or other third party material in this article are included in the article's Creative Commons license, unless indicated otherwise in a credit line to the material. If material is not included in the article's Creative Commons license and your intended use is not permitted by statutory regulation or exceeds the permitted use, you will need to obtain permission directly from the copyright holder. To view a copy of this license, visit http://creativecommons.org/licenses/by/4.0/.

(C) The Author(s) 2020 